\title{
Study of introgression lines of common wheat obtained with the participation of the synthetic forms T. miguschovae and Avrodes by allelic variants of the $W x$ genes
}

\author{
E.R. Davoyan ${ }^{1 *}$, R.O. Davoyan ${ }^{1}$, Yu.S. Zubanova ${ }^{1}$, D.S. Mikov ${ }^{1}$, D.M. Boldakov ${ }^{1}$, A.G. Chernook ${ }^{2}$, M.G. Divashuk ${ }^{2}$ \\ ${ }^{1}$ National Center of Grain of P.P. Lukyanenko, Krasnodar, Russia \\ ${ }^{2}$ St. All-Russia Research Institute of Agricultural Biotechnology, Moscow, Russia
}

DOI 10.18699/ICG-PlantGen2019-64

(c) Autors, 2019

* e-mail: davoyanro@mail.ru

\begin{abstract}
We studied the synthetic forms Triticum miguschovae (GGA $\left.{ }^{t} \mathrm{~A}^{\mathrm{D}} \mathrm{DD}\right)$ and Avrodes (BBAASS), as well as introgression lines of common wheat obtained with their participation from allelic variants of the $W x$ genes with the use of molecular markers. It was revealed that most of the lines carry wild-type alleles $W x-A 1 a, W x-B 1 a$ and $W x-D 1 a$, which were transferred from the recipient varieties. A fragment of amplification that is not typical of the $W x-B 1 a$ allele was found in line 393. The alleles of the $W x-A 1$ and $W x-B 1$ genes in the synthetic form of T. miguschovae are different from those in common wheat, and may have different phenotypic manifestations on the formation of starch. The lack of amplification from the $D$ genome in the synthetic form Avrodes is associated with the substitution of wheat chromosome 7D with its homoeologous chromosome from Ae. speltoides.

Key words: common wheat; synthetic forms; introgression lines; molecular markers; Wx-genes.
\end{abstract}

\section{Introduction}

The study of allelic variants of $W x$ genes is important for breeding aimed at creating varieties of bread wheat (Triticum aestivum L.) with a modified starch composition. The starch macromolecule consists of two types of glucose polymers: amylose and amylopectin. The ratio of amylose to amylopectin affects the properties of starch, which are the determining factors of its quality and end-use direction (Zeng et al., 1997). The enzyme responsible for the biosynthesis of amylose in wheat is the starch synthase GBSSI associated with granules encoded by the three homoeologous genes, $W x-A 1, W x-D 1$ and $W x-B 1$ (Nakamura et al., 1995). Each of these genes has several allelic variants. Wild-type alleles, $W x-A 1 a, W x-B 1 a$ and $W x-D 1 a$, do not carry mutations and actively express the GBSSI protein (Yamamori et al., 1994). Another type of allele is nonfunctional (null allele) and leads to a decrease in amylose content in starch. Polymorphism of allelic variants of $W x$ genes was studied for common and the hard wheat Triticum durum (Rodrígez-Quijano et al., 1998). As a result, it became possible to identify different alleles of the $W x$ genes, including null alleles, which were used as the basis for breeding programs aimed at producing wheat varieties with a modified starch composition (Nakamura et al., 1995). Recently, such studies have been carried out on wild relatives of common wheat (Guzman et al., 2011). In this regard, species of the genus Aegilops such as Aegilops tauschii Coss. (DD) and Aegilops speltoides Tausch. (SS) are of great interest, for they may be sources of new alleles of $W x$ genes with different enzymatic activity and have the potential to increase the genetic diversity of bread wheat for this trait. For transmission of common wheat valuable traits from wild relatives, the genome-substituted synthetic form Avrodes and the genome-added form T. miguschovae were used (Zhirov et al., 1984; Ivanov, 1984). When using these forms, introgression lines of common wheat were obtained, characterized by disease resistance, high protein content and other morphobiological features that are interesting for selection (Davoyan et al., 2012). Presumably, these lines can carry both known and new alleles of $W x$ genes.

\section{Materials and methods}

The objects of the study were the synthetic forms T. miguschovae and Avrodes, as well as 14 introgression lines of common wheat obtained with their participation. Commercial common wheat varieties Avrora, Mironovskaya 808, Echo, Bezostaya 1, Fisht, Grom, Vostorg, and Kavkaz were used as recipients. The synthetic forms and lines studied were obtained from the Department of Biotechnology of the National Center of Grain named after P.P. Lukyanenko. DNA isolation was performed by boiling in alkali. Evaluation of the lines for an allelic variant of $W x$ genes was performed with the use of PCR. Primers were selected on the basis of literature data, their names, sources and amplification conditions are presented in the Table 1.

\section{Results and discussion}

To study the samples for the allelic state of the $W x$ - $A 1$ gene, we used a code-marker created by Nakamura et al. (2002), which amplified fragments from all homeologous alleles of the $W x$ genes. In samples with wild-type alleles $W x-A 1 a$, amplification of two fragments, $410 \mathrm{bp}$ and $389 \mathrm{bp}$ in length, and in the presence of the null allele $W x-A 1 b, 410 \mathrm{bp}$ and $370 \mathrm{bp}$, is observed. In the majority of the lines studied, as well as in the synthetic form Avrodes, the wild-type allele $W x-A l a$ was identified (Figure 1). In line 393 obtained with the participation of T. miguschovae, heterogeneity was revealed, there is no amplification from the A genome in lane 8.2 (389-bp fragment). One bend was amplified with a different size from the 410-bp fragment in the synthetic form T. miguschovae, which is characteristic of common wheat, and also in line 393 there is no amplification from the $\mathrm{A}$ genome. 
Table 1

PCR conditions and primer names used to identify the corresponding alleles

\begin{tabular}{llll}
\hline Primers & Loci of genes (alleles) & Annealing temperature, ${ }^{\circ} \mathrm{C}$ & Source \\
\hline AFC/AR2 & $W x-A 1(A 1 b)$ & 65 & Nakamura et al., 2002 \\
4 F/4R & $W x-A 1, W x-B 1, W x-D 1$ & 58 & McLauchlan et al., 2001 \\
Wx-D1-2F/Wx-D1-2R & $W x-D 1(D 1 b)$ & 55 & Shariflou et al., 2001 \\
\hline
\end{tabular}

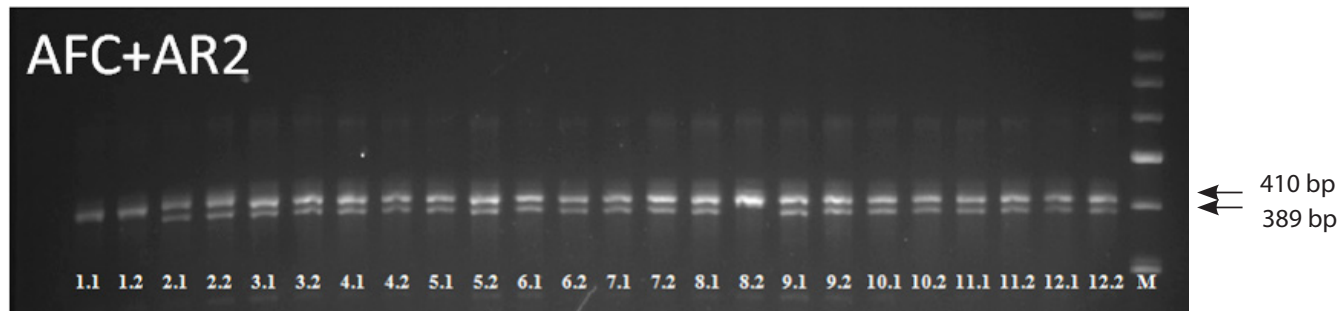

Figure 1. Electrophoregram of PCR products with primers AFC and AR2: 1: T. miguschovae; 2: Avrodes; 3: 241; 4: D16/1; 5: D16/2; 6: D16/4; 7: 1381; 8: 393; 9: 7; 10: 2269; 11: 1453; 12: variety 'Aurora'; M: marker length of DNA fragments.

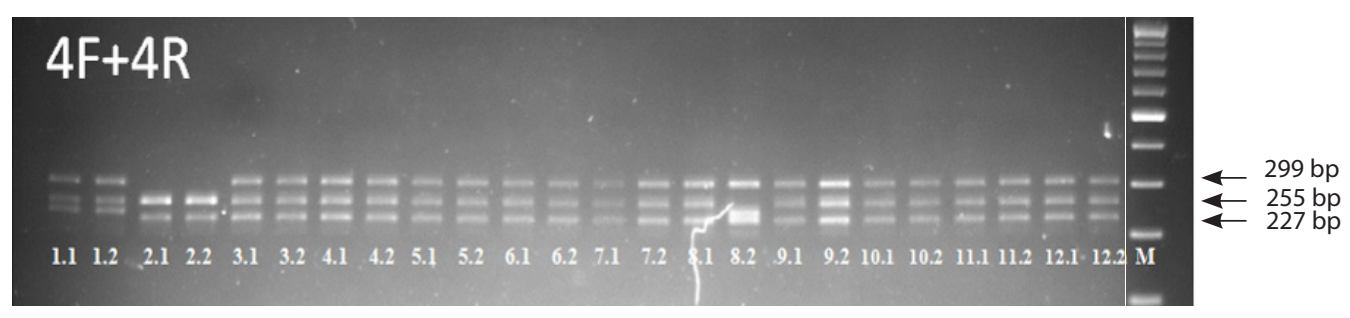

Figure 2. Electrophoregram of PCR products with primers $4 \mathrm{~F}$ and $4 \mathrm{R}$ 1: T. miguschovae; 2 : Avrodes; 3: 241; 4: D16/1; 5: D16/2; 6: D16/4; 7: 1381; 8: 393; 9: 7; 10:2269; 11: 1453; 12: variety Aurora; M: marker length of DNA fragments.

McLauchlan et al. (2001) developed a molecular labeling system for the $W x-B 1$ gene which amplify fragments from all three loci. Three DNA fragments $(299,255,227 \mathrm{bp})$ are amplified in samples with a wild-type allele. Two fragments (fragment of $227 \mathrm{bp}$ is absent) are synthesize in forms with a null allele (Figure 2). In the majority of the lines studied, the wild type allele $W x-B 1 a$ was detected. In the synthetic form T. miguschovae, the amplification fragment from the $\mathrm{B}$ genome differed in size from the 227-bp fragment characteristic of common wheat. Avrodes revealed no amplification from the D genome (299-bp amplification fragment). At the same time, the fragment characteristic of the genome B (257 bp) differed in intensity from the others. In the first case that was due to the replacement of the D genome of bread wheat with the $\mathrm{S}$ genome from Ae. speltoides and in the second, probably, due to double amplification from the B genome. Line 393 is heterogeneous, plants were identified with both amplification fragments typical of common wheat and different from it. So in Figure 2, in sample 8.2, there is no amplification from the A genome and an additional fragment is found which is similar in size to the fragment from the B genome (227 bp).

Identification of the allelic state of the $W x-D 1$ gene was carried out using a marker developed by Shariflou et al. (2001). This marker amplifies $279 \mathrm{bp}$ fragment in the case of null allele for the $W x-D 1$ gene; in the presence of the wildtype allele $-910 \mathrm{bp}$ fragment. A fragment specific for the wild-type allele $W x$-Dla was detected in the studied lines and in T. miguschovae. There was no amplification from the D genome in the synthetic form.

\section{Conclusions}

It was revealed that the majority of the lines studied present typical wild-type alleles for the common wheat genes $W x-A 1 a$, $W x-B 1 a$ and $W x-D 1 a$ as a result of the work carried out with the help of molecular markers. The wild-type alleles $W x-B 1 a$ and $W x-D 1 a$ were transmitted from the recipient varieties in the case of lines derived from Avrodes, and the Wx-Ala allele could be transmitted from both the recipient varieties and Avrodes. The $W x-A 1 a, W x-B 1 a$ alleles were transmitted from the recipient varieties in the lines obtained with the participation of T. miguschovae. Heterogeneity in line 393 is apparently related to the ongoing formative process. Probably, chromosome 7A from common wheat was replaced in this line by its homoeologous chromosome from T. miguschovae, hence no amplification from the A genome, and the reason why an additional fragment has been identified. The alleles of the $W x-A 1$ and $W x-B 1$ genes are different from those in common wheat in the synthetic form T. miguschovae, and may 
have a different phenotypic manifestation on the formation of starch. Thus, the selected molecular markers developed for identifying the null alleles of the $W x$ genes of bread wheat can also be used to identify the alleles of the $W x$ genes carried by T. miguschovae, Avrodes and the lines derived from them and for marker-assisted selection. The synthetic forms T. miguschovae and Avrodes, as well as line 393, carry alleles different from wild-type ones and are interesting for studying the polymorphism of allelic variants of $W x$ genes and their influence on the technological properties of wheat flour.

\section{References}

Davoyan R.O., Bebyakina I.V., Davoyan O.R., Zinchenko A.N., Davoyan E.R., Kravchenko A.M., Zubanova Yu.S. Synthetic forms as a basis for the preservation and use of the gene pool of wild relatives of common wheat. Vavilovsky Journal Genetics Breeding. 2012;16(1):44-51.

Zhirov E.G., Ternovskaya T.K. Genomic engineering in wheat. Vestnik Sel'skokhozyaistvennoi Nauki. 1984;10:58-66.

Ivanov G.I. New wheat amphidiploid with DDAbAb genomes. Scientific Techn. Bullet All-Russian Scientific-Research Institute Plant Industry. 1984;142:78-79.

Guzmán C., Caballero L., Alvarez J.B. Molecular characterization of the $W x-B 1$ allelic variants identified in cultivated emmer wheat and comparison with those of durum wheat. Mol. Breeding. 2011;28: 403-411.

McLauchlan A., Ogbonnaya F.C., Hollingsworth B. Development of robust PCR-based DNA markers for each homeoallele of granulebond starch synthase and their application in wheat breeding programs. Aust. J. Agric. Res. 2001;52:1409-1416.

Nakamura T., Jamamori M., Hirano H. Production of waxy (amylasefree) wheat. Mol. Gen. Genet. 1995;248:253-259.

Nakamura T., Vrinten P., Saito M., Konda M. Rapid classification of partial waxy wheats using PCR-based markers. Genome. 2002;45: $1150-1156$.

Rodriguez-Quijano M., Nieto-Taladriz M.T., Carrillo J.M. Polymorphism of waxy proteins in Iberian hexaploid wheats. Plant Breeding. 1998; 117:341-344.

Shariflou M.R., Hassani M.E., Sharp P.J. A PCR-based DNA marker for detection of mutant and normal alleles of the Wx-D1 gene of wheat. Plant Breeding. 2001;120(2):121-124.

Yamamori M., Nagamine T., Nakamura T., Endo T.R. Waxy protein deficiency and chromosomal location of coding genes in common wheat. Theoretical Applied Genetics. 1994;89(2-3):179-184.

Zeng M., Morris C.F., Batey I.I., Wrigley C.W. Sources of variation for starch gelatinization, pasting, and gelation properties in wheat. Cereal Chemistry. 1997;74:63-71.

Conflict of interest. The authors declare no conflict of interest. 